\title{
Secondary attack rate of pandemic influenza A(H1N1)2009 in Western Australian households, 29 May-7 August 2009
}

D Carcione (dale.carcione@health.wa.gov.au) ${ }^{1}$, C M Giele ${ }^{1}$, L S Goggin ${ }^{1}$, K SH Kwan ${ }^{1}$, D W Smith², G K Dowse ${ }^{1}$, D B Mak ${ }^{1}$, P Effler ${ }^{1}$

1. Communicable Disease Control Directorate, Department of Health, Perth, Western Australia, Australia

2. PathWest Laboratory Medicine WA, Nedlands, Western Australia, Australia

Understanding household transmission of the pandemic influenza $A\left(\mathrm{H}_{1} \mathrm{~N}_{1}\right) 2009$ virus, including risk factors for transmission, is important for refining public health strategies to reduce the burden of the disease. During the influenza season of 2009 we investigated transmission of the emerging virus in 595 households in which the index case was the first symptomatic case of influenza $A\left(\mathrm{H}_{1} \mathrm{~N}_{1}\right)_{2009}$. Secondary cases were defined as household contacts with influenzalike illness (ILI) or laboratory-confirmed influenza $A\left(H_{1} N_{1}\right)_{2009}$, occurring at least one day after but within seven days following symptom onset in the index case. ILI developed in 231 of the 1,589 household contacts, a secondary attack rate of $14.5 \%$ ( $95 \%$ confidence interval ( $\mathrm{Cl}): 12.9-16.4)$. At least one secondary case occurred in 166 of the 595 households (a household transmission rate of $27.9 \% ; 95 \% \mathrm{Cl}: 24.5-31.6$ ). Of these, $127(76.5 \%)$ households reported one secondary case and $39(23.5 \%)$ households reported two or more secondary cases. Secondary attack rates were highest in children younger than five years $(p=0.001)$, and young children were also more efficient transmitters $(p=0.01)$. Individual risk was not associated with household size. Prophylactic antiviral therapy was associated with reduced transmission $(p=0.03)$. The secondary attack rate of ILI in households with a confirmed pandemic influenza $A\left(\mathrm{H}_{1} \mathrm{~N}_{1}\right) 2009$ index case was comparable to that described previously for seasonal influenza.

\section{Introduction}

The world experienced the first influenza pandemic of the 21st century in 2009. Pandemic influenza $A\left(H_{1} N_{1}\right) 2009$ (hereafter to be referred to as pandemic influenza) was identified initially in Mexico and the United States (US) $[1,2]$ and spread rapidly to the southern hemisphere, becoming the dominant strain during the 2009 Australian winter [3]. In Western Australia (WA), pandemic influenza comprised over $90 \%$ of influenza notifications for which subtyping data were available. Pandemic influenza has since dominated the 2009/10 northern hemisphere winter and the 2010 southern hemisphere winter.

Understanding the transmission dynamics of pandemic influenza, including risk factors for transmission, is important in informing public health strategies to reduce the impact of the virus. Unfortunately, household transmission studies of the current [4-6], and previous influenza pandemics are scarce [7], and rely on studies of seasonal influenza [8-12]. Secondary attack rates reported for seasonal influenza range from 10\% to nearly $40 \%$ and vary with age, circulating strain, family composition, and levels of community exposure [8-12].

In the period between the notification of the first case in WA in late May 2009 and early August 2009 (before distribution of pandemic influenza vaccine), we investigated household transmission of pandemic influenza in WA. The objectives were to estimate the secondary attack rate and to describe the characteristics of index cases and their household contacts that were associated with risk of transmission.

\section{Methods}

Pandemic influenza index cases and their household contacts were recruited during a ten-week period encompassing the peak of pandemic influenza activity, from 29 May 2009 (four days after notification of the first confirmed case in WA), to 7 August 2009 [13]. Influenza is a notifiable disease in Australia, and cases were identified from the WA Notifiable Infectious Diseases Database, which is maintained by the Communicable Disease Control Directorate (CDCD). This database captures all notifiable disease reports for the State of WA, which has a population of over $\mathbf{2 . 2}$ million people [14]. All laboratory testing for pandemic influenza was carried out by PathWest Laboratory Medicine WA, a World Health Organization-designated National Influenza Centre. As a minimum, all specimens were tested by PCR directed at specific targets in the influenza A matrix gene and the pandemic influenza 
$\mathrm{H}_{1}$ haemagglutinin gene [15]. Over $90 \%$ of specimens were also tested for influenza $B$, and seasonal influenza $A\left(H_{1}\right)$ and $A\left(H_{3}\right)$ by PCR [15].

An index case was defined as anyone notified with pandemic influenza diagnosed by PCR during the study period and who otherwise met the eligibility criteria (see below). A household was defined as a group of two or more people living together in a domestic residence; residential institutions, such as boarding schools, hotels or prisons were excluded. A household contact was defined as any person who had resided in the same household as the index case for at least one night during the household exposure period (one day before to seven days after onset of illness in the index case). Index cases were excluded if they lived alone, did not spend time at the household after the onset of

\section{FIGURE 1}

Flow diagram of the investigation, household transmission study of pandemic influenza A(H1N1)2009, Western Australia, 29 May-7 August 2009

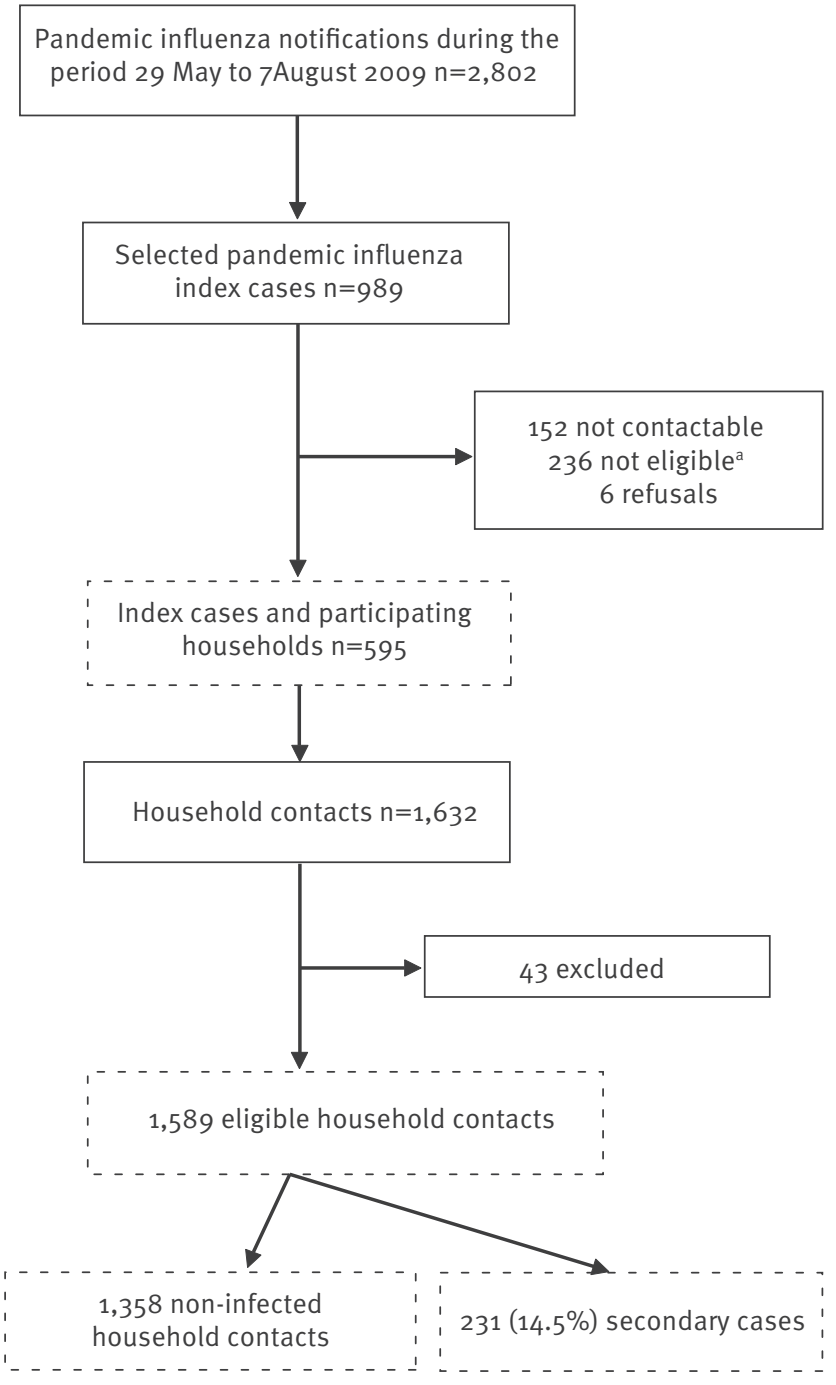

a Non-eligible index cases include: 140 who were not the first case of influenza-like illness in the household, 62 who lived alone, 28 who did not live at a private residential address, four who had a co-infection with another influenza virus, and two who could not communicate in English.

Dotted boxes denote those included in the final analysis. symptoms, had a co-infection with another influenza virus and/or were not the first symptomatic individual in the household. Household contacts who had the same symptom onset date as the index case, and were therefore possibly infected from the same source as the index case, were also excluded.

Influenza-like illness (ILI) was defined as fever $>38^{\circ} \mathrm{C}$, or a reliable history of fever of unknown temperature, AND cough and/or sore throat. A secondary case was defined as a household contact who developed an ILI or laboratory-confirmed influenza within seven days of symptom onset in the index case (distinctions were not made between secondary and tertiary cases in the household). Household transmission was deemed to have occurred if at least one household contact became a secondary case. Household contacts who did not develop an ILI or test positive for pandemic influenza were classified as uninfected household contacts. The secondary attack rate was calculated as the number of secondary cases divided by the total number of eligible household contacts. The mean serial interval was calculated from the sum of the time between the onset of ILI symptoms in all index and secondary case pairs.

Public health nurses interviewed each selected index case twice by telephone: within 48 hours of notification to $C D C D$ and the second time as close as possible to eight days after symptom onset. At the first interview, the reason for the investigation was explained and information was collected on: symptoms, use of antiviral medications, underlying medical conditions, vaccination for seasonal influenza and number of household contacts. The second interview collected information on household contacts, including: age, sex, number of days living in the household during the household exposure period, whether they shared the same room or bed as the index case, onset and symptoms of any illness during the exposure period, underlying medical conditions, use of antiviral prophylaxis, and vaccination for seasonal influenza. If an index case was unable to answer the questions or was under 18 years of age, an adult household member was interviewed as a proxy. A total of six attempts were made to contact the index case and/or household contacts, after which point they were deemed not contactable.

Information was sought on whether any household contacts had been notified with influenza in the exposure period by searching the notifications database for any confirmed influenza results matching the contact's name and date of birth with a specimen date within seven days of symptom onset. If no notification was recorded, PathWest Laboratory Medicine WA records were checked, to determine whether an influenza test had been performed and the result.

The secondary attack rate was analysed in relation to covariates measured at the index case and household contact levels using univariate chi-square test for proportions and t-tests for continuous variables. 
Subjects were stratified by age into pre-school-aged children ( $\leqslant 4$ years-old), school-aged children ( 5 to 18 years-old), 19 to 50 year-olds, and those aged over 50 years. Univariate odds ratios (OR) and 95\% confidence intervals $(\mathrm{Cl})$ were determined, and if multiple variables were found to be significant, they were entered as input for a backward step-wise logistic regression analysis. To adjust for clustering by household, generalised estimating equations were used to obtain $p$ values and confidence limits for ORs for all household contact analyses. All analyses were performed using PASW Version 17.0.2 (SPSS Inc., Chicago, IL). Information was collected as part of case follow-up for a notifiable disease of public health concern and did not require approval by a human research ethics committee.

\section{Results}

A total of 2,802 laboratory-confirmed pandemic influenza notifications were received during the ten-week study period. During the first six weeks, public health nurses attempted to contact each of the 468 pandemic influenza index cases notified in that period. Of those 468 notifications, $309(66.0 \%)$ were contacted, assessed eligible, and agreed to participate in the study. From 14 July to 7 August 2009, due to the increasing volume of notifications, a daily random sample of 20 pandemic influenza notifications per day were selected [16]. Of 521 additional index cases chosen by this method, 286 (54.9\%) were contactable and eligible for the study.

In total, $595(60.2 \%)$ of the 989 selected pandemic influenza index cases were eligible and participated in the investigation (Figure 1). Participating index cases were

\section{TABLE 1}

Characteristics of pandemic influenza A(H1N1)2009 index cases and their household contacts, Western Australia, 29 May-7 August $2009(\mathrm{n}=2,184)$

\begin{tabular}{|c|c|c|}
\hline Characteristic & $\begin{array}{l}\text { Pandemic influenza index cases }{ }^{\mathrm{a}} \\
\qquad \mathrm{N}^{\mathrm{b}}=595\end{array}$ & $\begin{array}{l}\text { Household contacts } \\
\qquad \mathrm{N}^{\mathrm{b}}=1,589\end{array}$ \\
\hline Age, mean (standard deviation) & $25.7(16.4)$ & $30.1(18.8)$ \\
\hline Age range, years & $0-79$ & $0-103$ \\
\hline \multicolumn{3}{|l|}{ Age group } \\
\hline $0-4$ years & $26(4.4)$ & $124(7.8)$ \\
\hline $5-18$ years & $237(39.8)$ & $447(28.1)$ \\
\hline $19-50$ years & $277(46.6)$ & $757(47.6)$ \\
\hline$\geq 51$ years & $55(9.2)$ & $228(14 \cdot 3)$ \\
\hline \multicolumn{3}{|l|}{ Sex } \\
\hline Male & $294(49.4)$ & $806(50.7)$ \\
\hline Female & $301(50.6)$ & $783(49.3)$ \\
\hline \multicolumn{3}{|l|}{ Indigenous status } \\
\hline Aboriginal & $34(5.7)$ & $62(3.9)$ \\
\hline \multicolumn{3}{|l|}{ Underlying medical conditions } \\
\hline Diabetes & $35(5.9)$ & $35(2.2)$ \\
\hline Heart disease & $19(3.2)$ & $33(2.1)$ \\
\hline Respiratory disease & $116(19.5)$ & $126(7.9)$ \\
\hline Renal disease & $2(0.3)$ & $5(0.3)$ \\
\hline Neurological disease & $4(0.7)$ & $13(0.8)$ \\
\hline Haematological disorder & $11(1.8)$ & $11(0.7)$ \\
\hline Metabolic disease (excluding diabetes) & $9(1.5)$ & $2(0.1)$ \\
\hline Immune impairment & $15(2.5)$ & $19(1.2)$ \\
\hline Morbid obesity & $41(6.9)$ & $60(3.8)$ \\
\hline Current smoker & $58(9.7)$ & $137(8.6)$ \\
\hline Pregnant (females only) & $20(3.4)$ & $13(1.7)$ \\
\hline Any underlying condition ${ }^{c}$ & $232(39.0)$ & $270(17.0)$ \\
\hline \multicolumn{3}{|l|}{ Antivirals } \\
\hline Yes & $238(40.0)$ & $220(13.8)$ \\
\hline $\mathrm{No}^{\mathrm{d}}$ & $331(55.6)$ & $1,327(83.5)$ \\
\hline \multicolumn{3}{|l|}{ Seasonal influenza vaccination in 2009} \\
\hline Yes & $125(25.0)$ & $304(19.1)$ \\
\hline No & $394(66.2)$ & $1,162(73.1)$ \\
\hline
\end{tabular}

${ }^{a}$ Number of people (percentage), unless otherwise indicated.

${ }^{\mathrm{b}}$ Respondents may not add up to total because of missing information for some variables.

c Patient reported at least one of the underlying medical conditions listed.

${ }^{d}$ Refers to treatment use of antiviral drugs in index cases and preventative use of antiviral drugs in household contacts. 
very similar with respect to age (median age 25 years) and sex, to all remaining pandemic influenza cases who were notified in the study period and who were not interviewed or eligible to participate $(n=2,207)$.

\section{TABLE 2}

Characteristics of the household contacts of influenza A(H1N1)2009 index cases and secondary attack rates associated with these characteristics, Western Australia, 29 May-7 August $2009(n=1,589)$

\begin{tabular}{|c|c|c|c|c|}
\hline Characteristic of household contact & $\begin{array}{l}\text { Number of household contacts } \\
\qquad n^{a}=1,589\end{array}$ & Secondary attack rate, $\%$ & Odds ratio $(95 \% \mathrm{Cl})$ & $\mathrm{p}$ value \\
\hline \multicolumn{5}{|l|}{ Age } \\
\hline $0-4$ years & 124 & 22.6 & $3.40(1.80$ to 6.45$)$ & \\
\hline $5-18$ years & 447 & 17.2 & 2.43 (1.41 to 4.17$)$ & $0.001^{\mathrm{b}}$ \\
\hline $19-50$ years & 757 & 13.7 & $1.86(1.10$ to 3.14$)$ & \\
\hline$\geq 51$ years & 228 & 7.9 & 1.00 & \\
\hline \multicolumn{5}{|l|}{ Sex } \\
\hline Male & 806 & 14.6 & 1.04 (0.79 to 1.37 ) & 0.80 \\
\hline Female & 783 & $14 \cdot 3$ & 1.00 & \\
\hline \multicolumn{5}{|l|}{ Indigenous status } \\
\hline Aboriginal & 62 & 8.1 & $0.49(0.20$ to 1.24$)$ & 0.13 \\
\hline Non-Aboriginal & 1,474 & 15.1 & 1.00 & \\
\hline \multicolumn{5}{|l|}{ Present for the entire index illness } \\
\hline Yes & 1497 & $14 \cdot 9$ & 2.49 (0.99 to 6.22) & 0.05 \\
\hline No & 76 & 6.6 & 1.00 & \\
\hline \multicolumn{5}{|l|}{ Shared the same room as the index } \\
\hline Yes & 337 & 16.6 & 1.24 (o.89 to 1.72$)$ & 0.20 \\
\hline No & 1226 & 13.9 & 1.00 & \\
\hline \multicolumn{5}{|l|}{ Shared the same bed as the index } \\
\hline Yes & 289 & 17.6 & 1.35 (0.96 to 1.90$)$ & 0.09 \\
\hline No & 1275 & 13.7 & 1.00 & \\
\hline \multicolumn{5}{|l|}{ Underlying medical conditions ${ }^{c}$} \\
\hline Diabetes & 35 & 8.6 & 0.54 (0.16 to 1.78$)$ & 0.31 \\
\hline Heart disease & 33 & 15.2 & 1.04 (0.40 to 2.73$)$ & 0.93 \\
\hline Respiratory disease & 126 & 22.2 & $1.76(1.13$ to 2.75$)$ & 0.01 \\
\hline Renal disease & 5 & 20.0 & 1.46 (0.16 to 13.12) & 0.74 \\
\hline Neurological disease & 13 & 23.1 & $1.76(0.48$ to 6.44$)$ & 0.39 \\
\hline Haematological disorder & 11 & 0.0 & - & 0.17 \\
\hline Metabolic disease (excluding diabetes) & 2 & 0.0 & - & 0.56 \\
\hline Immune impairment & 19 & 21.1 & $1.57(0.52$ to 4.78$)$ & 0.43 \\
\hline Morbid obesity & 60 & 16.7 & $1.17(0.59$ to 2.35$)$ & 0.65 \\
\hline Current smoker & 137 & 10.2 & $0.64(0.36$ to 1.14$)$ & 0.13 \\
\hline Pregnant (females only) & 13 & 0.0 & - & 0.22 \\
\hline Any underlying condition ${ }^{d}$ & 270 & 18.5 & 1.40 (0.99 to 1.98$)$ & 0.06 \\
\hline \multicolumn{5}{|l|}{ Prophylactic antiviral therapy } \\
\hline Yes & 220 & 9.5 & $0.58(0.36$ to 0.94$)$ & 0.03 \\
\hline No & 1,327 & $15 \cdot 3$ & 1.00 & \\
\hline \multicolumn{5}{|l|}{ Seasonal influenza vaccination in 2009} \\
\hline Yes & 304 & 15.1 & $1.01(0.71$ to 1.44$)$ & 0.95 \\
\hline No & 1,162 & 15.0 & 1.00 & \\
\hline \multicolumn{5}{|l|}{ Household size } \\
\hline 2 persons & 135 & 16.3 & $1 \cdot 00$ & \\
\hline 3 persons & 273 & 12.5 & $0.73(0.41$ to 1.31$)$ & $0.65^{b}$ \\
\hline 4 persons & 514 & 14.2 & $0.85(0.51$ to 1.43$)$ & \\
\hline$\geq 5$ persons & 667 & $15 \cdot 3$ & $1.01(0.59$ to 1.73$)$ & \\
\hline
\end{tabular}

${ }^{a}$ Respondents may not add up to total because of missing information for some variables.

${ }^{b}$ Chi-square test for trend.

'Odds ratio for individual underlying medical conditions is the odds of infection among contacts with that condition, versus the odds in those not reporting that condition.

d Patient reported at least one of the underlying medical conditions listed.

Variables in blue were statistically significant and were included in the multivariate logistic regression. 
There were 1,632 household contacts in the 595 participating households. Forty-three contacts were excluded, 14 with insufficient information and 29 who became ill on the same day as the index case, leaving 1,589 household contacts for the final analysis (Figure 1). Characteristics of index cases and household contacts are shown in Table 1. Index cases were younger, and more likely to report underlying medical conditions and to have had seasonal influenza vaccine, than the household contacts.

Overall, 231 secondary cases occurred among the 1,589 household contacts, giving a secondary attack rate of 14.5\% (95\% Cl: 12.9-16.4). The secondary attack rate in households without co-primary household contacts $(n=570)$ was similar to that in all households including those with co-primary contacts $(13.6 \%$ and $14.5 \%$, respectively, $p=0.47$ ).

In order to estimate the proportion of ILI cases due to pandemic influenza, we identified all secondary cases who had swabs collected within 48 hours of onset of ILI symptoms, at which time the yield should be optimal [17]. Among these 29 cases, 27 were PCR-positive for pandemic influenza, suggesting ILI was highly predictive of pandemic influenza infection in these households.

\section{FIGURE 2}

Secondary attack rate of influenza A(H1N1)2009 index cases and household contacts, by age group, Western Australia, 29 May-7 August $2009(\mathrm{n}=2,184)$

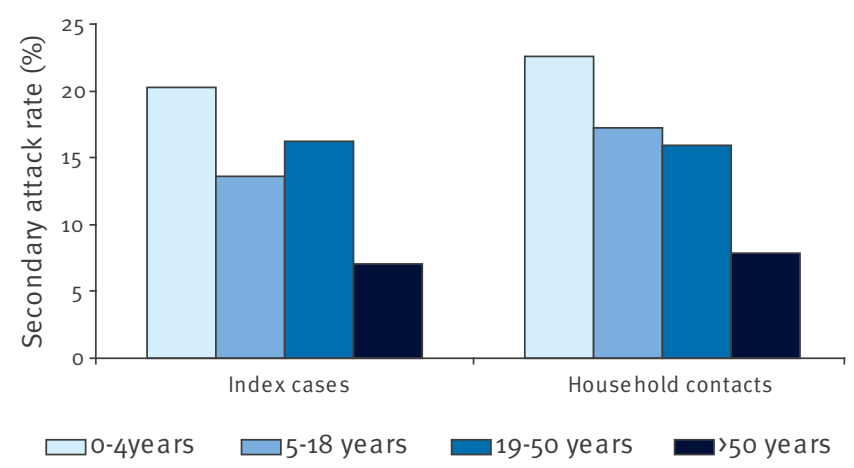

\section{FIGURE 3}

Distribution of days (serial interval) from onset of illness in the index case to onset of influenza-like illness in the secondary case(s), Western Australia, 29 May-7 August $2010(\mathrm{n}=231)$

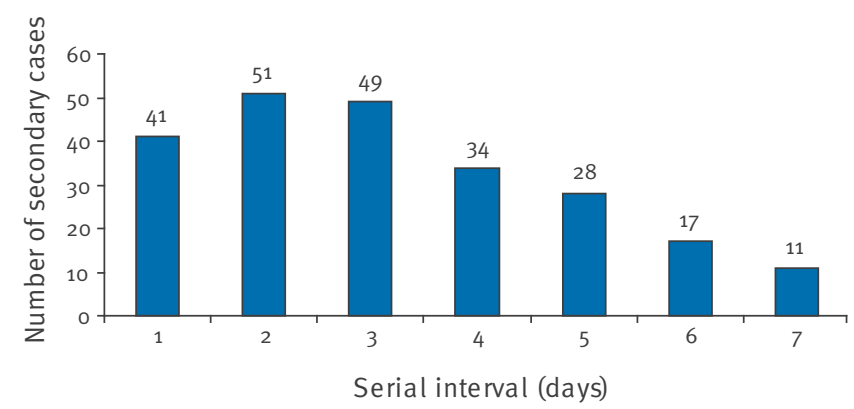

One or more secondary cases occurred in 166 of the 595 households (27.9\%; $95 \% \mathrm{Cl}: 24.5-31.6)$. Of the 166 households with secondary cases $127(76.5 \%)$ reported one case, $20(12.0 \%)$ reported two, $13(7.8 \%)$ reported three, five $(3.0 \%)$ reported four, and one $(0.6 \%)$ reported five secondary cases.

Table 2 shows the characteristics of the household contacts and secondary attack rates associated with these characteristics. Secondary cases (mean age 25.2 years) were significantly ( $p<0.001$ ) younger than uninfected household contacts (mean age 31.0 years). There was a clear inverse association between age and secondary attack rate $(p=0.001)$, with the odds of illness 3.4 times higher in o to 4-year-old children compared to adults aged 51 years or older. Secondary attack rates were elevated in household contacts who were present for the entire household exposure period, although this just failed to reach statistical significance $(O R=2.49, p=0.05)$. Among a range of underlying medical conditions, only respiratory disease (including asthma) was significantly more prevalent in secondary cases $(O R=1.72, p=0.01)$ compared to uninfected contacts. Uninfected contacts were more likely to have taken antiviral prophylaxis (14.7\%) compared to secondary cases $(9.1 \% ; p=0.03)$. Transmission was not associated with sex, indigenous status, smoking, sharing a room or bed with the index case, household size or 2009 seasonal influenza vaccination status of household contacts. In the multivariate logistic regression model, which included age ( $p<0.001)$, respiratory disease $(p=0.031)$ and prophylactic antiviral therapy $(p=0.031)$, all remained independent predictors for (or against, in the case of prophylactic antiviral therapy) becoming a secondary case.

As illustrated in Figure 2, there was an inverse association between secondary attack rates and age of both index cases and household contacts. Young index cases were more likely to transmit infection to their household contacts, and young household contacts were more likely to be infected.

Amongst the range of symptoms reported by index cases, the following resulted in significantly more transmission to secondary cases than others: cough $(p=0.04)$, shortness of breath ( $p<0.001)$, fatigue ( $p<0.001)$, myalgia $(p=0.009)$, rigors $(p=0.003)$, diarrhoea $(p=0.001)$ and vomiting ( $p<0.001)$. There was no difference in the secondary attack rate associated with index cases who had taken antiviral treatment (14.9\%) compared to those who had not $(14.1 \%, p=0.70)$. The mean interval from onset of illness to treatment of the index case was three days and the median interval was two days.

The median serial interval was 3.0 days (range: 1-7 days) and the mean serial interval was 3.2 days (Figure $3)$. Of the 28 secondary cases occurring six to seven days after the index case, 10 occurred in households with two or more secondary cases. The median and 
mean serial intervals were unchanged if households with more than one secondary case (i.e. possible tertiary cases) were excluded.

\section{Discussion}

This investigation found that the secondary attack rate of ILI among household contacts of a confirmed pandemic influenza index case in Western Australia was $14.5 \%$, and that household transmission (to at least one secondary case) occurred in $\mathbf{2 7 . 9 \%}$ of households.

Some studies on pandemic influenza and seasonal influenza $A\left(\mathrm{H}_{1} \mathrm{~N}_{1}\right)$ epidemics have estimated considerably higher secondary attack rates. A US modelling study based on case clusters early in the 2009 influenza pandemic, estimated the risk of ILI in household contacts of pandemic influenza index cases to be $\mathbf{2 7 . 3} \%$ [18]. Similarly, the secondary attack rate of laboratory-confirmed pandemic influenza cases in Kenya between June and July 2009, prior to the use of antiviral drugs, was $26.0 \%$ [19] and in a recently published Canadian study of 42 households reached as high as $45 \%$ [5]. In the $1978-1979$ influenza $A\left(\mathrm{H}_{1} \mathrm{~N}_{1}\right)$ seasonal epidemic, the US had an estimated secondary attack rate of $30.6 \%$ [9]. There are no estimates of transmissibility within households for the 1918-1919 influenza $\mathrm{A}\left(\mathrm{H}_{1} \mathrm{~N}_{1}\right)$ pandemic.

However, other studies report much lower rates, with one study in an English boarding school estimating a $5.4 \%$ to $11.9 \%$ secondary attack rate for ILI, depending on the school year [20]. Epidemiological field studies undertaken in several states of the US during the initial wave of 2009 pandemic influenza found secondary attack rates of ILI ranging from $8 \%$ to $12 \%$ in household contacts of those with ILI [21], and in more recently published US studies the household secondary attack rate associated with index cases of pandemic influenza 2009 was 13\% for acute respiratory illness, and ranged from $9-10 \%$ for ILI $[4,6]$. The secondary attack rates from these studies of pandemic influenza are comparable to the one we observed in WA. The slightly higher secondary attack rates of ILI in WA may reflect the greater intensity of a winter pandemic season compared to the late spring season experienced in the initial northern hemisphere pandemic wave.

Transmission was highest in households with an index case of pre-school age. Although a recent US study found children with pandemic influenza to be no more infectious than adults [4], our findings are consistent with the many other studies that have shown increased transmission from children in both households and communities. This is presumably because children shed larger amounts of influenza virus and for longer periods of time than adults, are less conscious of hygiene and require more close contact $[9,12,22-26]$. In addition, children have been found to be the main source of influenza in households during interpandemic seasons $[9,12]$.
Other characteristics of pandemic influenza index cases that were significantly associated with transmission in households included the symptoms cough, shortness of breath, fatigue, myalgia, rigors, diarrhoea, and vomiting. These symptoms were possibly markers of more serious illness which was associated with higher or more prolonged virus shedding, and/or required closer and more prolonged contact with their carers. The lack of a statistically significant effect of fever or other respiratory symptoms such as sore throat and runny nose on infectivity of pandemic influenza is similar to the findings in the above-mentioned US study in 2009 [4].

In our investigation household contacts of pre-school age had the highest secondary attack rate (22.6\%), and adults aged 51 years and older the lowest (7.9\%). This is similar to the secondary attack rates reported during the pandemic influenza season in the US in late spring 2009 [4,6]. Children, in particular those who attend day care or school, are considered to be at high risk of influenza infection, with attack rates ranging from $20 \%$ to $50 \%$ during seasonal interpandemic years [23$25,27]$. The low secondary attack rates in household contacts aged over 50 years is consistent with the relatively low incidence of pandemic influenza 2009 in older adults that has been attributed to cross-protection against the pandemic virus following exposure to influenza $A\left(\mathrm{H}_{1} \mathrm{~N}_{1}\right)$ viruses early in life $[28,29]$.

Treatment of index cases with the antiviral drug oseltamivir did not reduce transmission in households, possibly because it was given late, as indicated by the mean interval of three days between onset of illness in the index case and treatment. Conversely, secondary attack rates among household contacts who had received a prophylactic course of oseltamivir was significantly lower than in those who had not $(9.5 \%$ versus $15.3 \%$ ), consistent with its reported efficacy for prevention of pandemic [30] and seasonal influenza household transmission [31,32]. A study in Japan in mid-2009 showed an even more dramatic difference in secondary attack rates among household contacts who did not receive prophylaxis compared to those who did (7.6\% versus $0.8 \%$ ), although this could be biased by the mass use of chemoprophylaxis in the community [30]. Our results provide support for the recommendation for early antiviral use as a preventive measure for close contacts during a pandemic, notwithstanding the need to consider that recommendation in the context of parameters such as the severity of illness attributable to the pandemic virus, the stage of the pandemic response, possible adverse effects, emergence of resistant strains, and the cost and feasibility of widespread use of antiviral prophylaxis.

Household contacts with an underlying respiratory disease were independently associated with becoming a secondary case. It is possible that people with underlying respiratory disease are no more likely to become infected, but are more likely to become symp- 
tomatic when infected with influenza and therefore to be identified as a secondary case.

Interestingly, household size was not associated with individual risk of secondary infection in household contacts. The same was observed in a French study [33]. By contrast, a recent US study found an inverse association between secondary attack rate and household size [4], highlighting the need for further investigation and the consideration of data from different geographical and cultural backgrounds when determining transmission dynamics.

Estimates of the mean serial interval for seasonal influenza from empirical data range from two to four days $[11,34]$, and different estimates of the mean serial interval of the 2009 pandemic influenza, using both empirical and modelling data, were 2.5 to 2.7 days $[35,36]$, 2.6 to 2.9 days [4], and 3.2 days [18]. Our empirical estimate of the serial interval of pandemic influenza in WA households, 3.2 days, matches these results closely.

Our investigation has a number of strengths and limitations. Whilst we did not include all confirmed pandemic influenza cases in WA, the sample size was large and representative of all laboratory-confirmed pandemic cases (although we were unable to control for biases stemming from who was tested and who was not) during the study period. Data were collected from nearly all participants within seven days of notification, increasing the likelihood of accurate recall of information. While a number of index cases were unable to answer the questions and an adult proxy answered questions on their behalf, this was unlikely to introduce any systematic bias, and if anything would be expected to weaken any real associations.

The fact that the household contacts who reported ILI were not all tested for influenza infection may have resulted in an overestimation of the number of secondary cases actually attributed to pandemic influenza. However, of the secondary cases who did undergo testing within 48 hours of symptom onset, the majority (27 of 29) were confirmed to have pandemic influenza infection. This estimate may be biased upwards by preferential testing of those with influenza, as they may have had more severe clinical illness than individuals whose ILI had other causes.

It is also possible that secondary cases occurred as a result of exposure outside the household. However, a study of the molecular epidemiology of seasonal influenza $A$ virus transmission found that the majority of cases of influenza in a household were the result of transmission from the household index case and not from external community sources [37].

This was a unique opportunity to study transmission of pandemic influenza within households at a time when little information on the disease was available. This large-scale investigation has shown that secondary attack rates were similar to those seen with seasonal influenza, as was the estimated serial interval. While the secondary attack rate for children at pre-school age was within the lower range of published rates for interpandemic seasonal influenza, young children still had the highest attack rates of all age groups, and infected index children were more likely to transmit infection. The results also indicate household contacts with a respiratory disease are at an increased risk of becoming secondary cases. In a pandemic setting where antiviral medications are in short supply, it may be important to prioritise the provision of prophylaxis to the young and those with specific underlying medical conditions, such as respiratory disease, so as to optimise the likelihood of reducing the individual, family and community burden of disease.

\section{Acknowledgements}

We would like to thank Megan Scully, Vince Rettura and the public health nurses who helped coordinate and carry out the telephone interviews. We would also like to thank Simon Williams from the PathWest Laboratory Medicine WA for providing testing and sub-typing data.

Funding and support was provided by The Department of Health, Western Australia.

\section{References}

1. Centres for Disease Control (CDC). Swine influenza $A\left(\mathrm{H}_{1} \mathrm{~N}_{1}\right)$ infection in two children - southern California, March-April 2009. MMWR Morb Mortal Wkly Rep. 2009;58(15):400-2.

2. Novel Swine-Origin Influenza $A\left(\mathrm{H}_{1} \mathrm{~N}_{1}\right)$ Virus Investigation Team, Dawood FS, Jain S, Finelli L, Shaw MW, Lindstrom S, et al. Emergence of a novel swine-origin influenza $A\left(\mathrm{H}_{1} \mathrm{~N}_{1}\right)$ virus in humans. N Engl J Med. 2009;360(25):2605-15.

3. Bishop JF, Murnane MP, Owen R. Australia's winter with the 2009 pandemic influenza $A\left(\mathrm{H}_{1} \mathrm{~N}_{1}\right)$ virus. N Engl J Med. 2009;361(27):2591-4

4. Cauchemez S, Donnelly CA, Reed C, Ghani, AC, Fraser C, Kent CK, et al. Household transmission of 2009 pandemic influenza $A\left(\mathrm{H}_{1} \mathrm{~N}_{1}\right)$ virus in the United States. N Engl J Med. 2009;361(27):2619-27.

5. Papenburg J, Baz M, Hamelin M, Rheaume C, Carbonneau J, Ouakki M, et al. Household transmission of the 2009 pandemic $\mathrm{A} / \mathrm{H}_{1} \mathrm{~N}_{1}$ influenza virus: elevated laboratory-confirmed secondary attack rates and evidence of asymptomatic infections. Clin Infect Dis. 2010;51(9):1033-41.

6. Morgan OW, Parks S, Shim T, Blevins PA, Lucas PM, Sanchez $\mathrm{R}$, et al. Household transmission of pandemic ( $\left.\mathrm{H}_{1} \mathrm{~N}_{1}\right) 2009$, San Antonio, Texas, USA, April-May 2009. Emerg Infect Dis. 2010;16(4):631-7.

7. Nishiura H, Chowell G. Household and community transmission of the Asian influenza $A\left(\mathrm{H}_{2} \mathrm{~N}_{2}\right)$ and influenza $B$ viruses in 1957 and 1961. Southeast Asian J Trop Med Public Health. 2007;38(6):1075-83.

8. Carrat F, Sahler C, Rogez S, Leruez-Ville M, Freymuth F, Le Gales C, et al. Influenza burden of illness: estimates from a national prospective survey of household contacts in France. Arch Intern Med. 2002;162(16):1842-8.

9. Longini IM Jr, Koopman J, Monto AS, Fox JP. Estimating household and community transmission parameters for influenza. Am J Epidemiol. 1982;115(5):736-51.

10. Longini IM Jr, Koopman JS, Haber M, Cotsonis GA. Statistical inference for infectious diseases. Risk-specific household and community transmission parameters. Am J Epidemiol. 1988;128(4):845-59.

11. Vibound C, Boëlle P, Cauchemez S, Lavenu A, Valleron AJ, Flahault $A$, et al. Risk factors of influenza transmission in household. Br J Gen Pract. 2004;54(506):684-9. 
12. Fox JP, Hall CE, Cooney MK, Foy HM. Influenza virus infections in Seattle families, 1975-1979: study design, methods, and the occurrence of infections by time and age. Am J Epidemiol. 1982;116(2):212-27.

13. First case of Swine Flu in WA. $\mathrm{H}_{1} \mathrm{~N}_{1}$ Influenza og latest news. Canberra: Australian Government Department of Health and Ageing; 25 May 2009. Available from: http:// www.healthemergency.gov.au/internet/healthemergency/ publishing.nsf/Content/news-028

14. Population growth. Australian Bureau of Statistics, $1367.5-$ Western Australian Statistical Indicators, Sep 2009. [Accessed 12 December 2009]. Available from: http://www.abs.gov.au/ ausstats/abs@.nsf/o/C9281FB5A31B19F6CA25765Bo01232FF? opendocument

15. Chidlow G, Harnett G, Williams S, Levy A, Speers D, Smith DW. Duplex real-time RT-PCR assays for the rapid detection and identification of pandemic ( $\left.\mathrm{H}_{1} \mathrm{~N}_{1}\right) 2009$ and seasonal influenza viruses $\mathrm{A} / \mathrm{H}_{1}, \mathrm{~A} / \mathrm{H}_{3}$ and $\mathrm{B}$. J Clin Microbiol. 2010;48(3):862-6.

16. Carcione D, Giele C, Dowse GK, Mak DB, Goggin L, Kwan K, et al. Comparison of pandemic $\left(\mathrm{H}_{1} \mathrm{~N}_{1}\right) 2009$ and seasonal influenza, Western Australia, 2009. Emerg Infect Dis. 2010;16(9):1388-95.

17. Dwyer DE, Smith DW, Catton MG, Barr IG. Laboratory diagnosis of human seasonal and pandemic influenza virus infection. Med J Aust. 2006;185(10 Suppl):S48-S53.

18. Yang Y, Sugimoto JD, Halloran ME, Basta NE, Chao DL, Matrajt $L$, et al. The transmissibility and control of pandemic influenza A ( $\left.\mathrm{H}_{1} \mathrm{~N}_{1}\right)$ virus. Science. 2009;326(5953):729-33.

19. Centers for Disease Control and Prevention (CDC).Introduction and transmission of 2009 pandemic influenza $A\left(\mathrm{H}_{1} \mathrm{~N}_{1}\right)$ Virus - Kenya, June-July 2009. MMWR Morb Mortal Wkly Rep. 2009;58(41):1143-6.

20. Smith A, Coles S, Johnson S, Saldana L, Ihekweazu C, O'Moore E. An outbreak of influenza A ( $\left.\mathrm{H}_{1} \mathrm{~N}_{1}\right) \mathrm{v}$ in a boarding school in South East England, May-June 2009. Euro Surveill. 2009;14(27):pii=19263. Available from: http://www. eurosurveillance.org/ViewArticle.aspx?Articleld=19263

21. $2009 \mathrm{H}_{1} \mathrm{~N}_{1}$ early outbreak and disease characteristics, October 27, 2009. Atlanta: Centres for Disease Control and Prevention. [Accessed 12 December 2009] Available from: http://www.cdc. gov/h1n1flu/surveillanceqa.htm

22. Neuzil KM, Hohlbein C, Zhu Y. Illness among schoolchildren during influenza season: effect on school absenteeism, parental absenteeism from work, and secondary illness in families. Arch Pediatr Adolesc Med. 2002;156(10):986-91.

23. Long CE, Hall CB, Cunningham CK, Weiner LB, Alger KP, Gouveia $M$, et al. Influenza surveillance in communitydwelling elderly compared with children. Arch Fam Med. 1997;6(5):459-65.

24. Neuzil KM, Zhu Y, Griffin MR, Edwards KM, Thompson JM, Tollefson SI, et al. Burden of interpandemic influenza in children younger than 5 years: a 25 -year prospective study. J Infect Dis. 2002;185(2):147-52.

25. Principi N, Esposito S, Marchisio P, Gasparini R, Crovari P. Socioeconomic impact of influenza in healthy children and their families. Pediatr Infect Dis J. 2003;22:(S10):S207-10.

26. Reichert TA, Sugaya N, Fedson DS, Glezen WP, Simonsen L, Tashiro M. The Japanese experience with vaccinating schoolchildren against influenza. N Eng J Med. 2001;344(12):889-96.

27. Hurwitz ES, Haber M, Chang A, Shope T, Teo S, Ginsberg M, et al. Effectiveness of influenza vaccination of day care children in reducing influenza-related morbidity among household contacts. JAMA. 2000;284(13):1677-82.

28. Fisman DN, Savage R, Gubbay J, Achonu C, Akwar H, Farrell DJ, et al. Older age and a reduced likelihood of $2009 \mathrm{H} 1 \mathrm{~N}_{1}$ virus infection. N Engl J Med. 2009;361(20):2000-1.

29. Hancock K, Veguilla V, Lu X, Zhong W, Butler EN, Sun H, et al. Cross-reactive antibody responses to the 2009 pandemic $\mathrm{H}_{1} \mathrm{~N}_{1}$ influenza virus. N Engl J Med. 2009;361(20):1945-52

30. Odaira F, Takahashi H, Toyokawa T, Tsuchihashi Y, Kodama T, Yahata Y, et al. Assessment of secondary attack rate and effectiveness of antiviral prophylaxis among household contacts in an influenza $A\left(\mathrm{H}_{1} \mathrm{~N}_{1}\right)$ v outbreak in Kobe, Japan, May-June 2009. Euro Surveill 2009;14(35):pii=19320. Available from: http://www.eurosurveillance.org/ViewArticle. aspx?Articleld $=19320$

31. Welliver R, Monto AS, Carewicz O, Schatteman E, Hassman $M$, Hedrick J, et al. Effectiveness of oseltamivir in preventing influenza in household contacts: a randomized control trial. JAMA. 2001;285(6):748-54.

32. Hayden FG, Belshe R, Villanueva C, Lanno R, Hughes C, Small I, et al. Management of influenza in households: a prospective randomised comparison of oseltamivir treatment with or without prophylaxis. J Infect Dis. 2004;189(3):440-9.
33. Cauchemez S, Carrat F, Vibound C, Valleron AJ, Boëlle PY. A Bayesian MCMC approach to study transmission of influenza: application to household longitudinal data. Stat Med .2004;23(22):3469-87.

34. Cowling BJ, Fang VJ, Riley S, Malik Peiris JS, Leung GM. Estimation of the serial interval of influenza. Epidemiology. 2009;20(3):344-7.

35. White LF, Wallinga J, Finelli L, Reed C, Riley S, Lipsitch M, et al. Estimation of the reproductive number and the serial interval in early phase of the 2009 influenza $\mathrm{A} / \mathrm{H}_{1} \mathrm{~N}_{1}$ pandemic in the USA. Influenza Other Respi Viruses. 2009;3(6):267-76.

36. Lessler J, Reich NG, Cummings DA, the New York City Department of Health and Mental Hygiene Swine Influenza Investigation Team, Nair HP, Jordan HT, et al. Outbreak of 2009 pandemic influenza $A\left(\mathrm{H}_{1} \mathrm{~N}_{1}\right)$ at a New York City school. N Eng J Med. 2009;361(27):2628-36.

37. Gubareva LV, Novikov DV, Hayden FG. Assessment of hemagglutinin sequence heterogeneity during influenza virus transmission in families. J Infect Dis. 2002;186(11):1575-81. 